\title{
Reinserción social y laboral de inmigrantes retornados de Estados Unidos en un contexto urbano*
}

\section{Social and labor reintegration of immigrants returned from the United States in an urban context}

\author{
Liliana Rivera Sánchez** \\ Centro de Estudios Sociológicos de El Colegio de México, Ciudad de México, México \\ ISSN: ISSN-OI85-4259; e- ISSN: 2007-9I76 \\ ORCID: https://orcid.org/0000-0003-005I-I76X \\ DoI: http://dx.doi.org/I0.28928/ri/752013/atc2/riverasanchezl
}

\section{Resumen}

En el presente artículo se examinan el proceso y las condiciones contemporáneas del retorno de migrantes mexicanos de Estados Unidos en contextos urbanos, con el propósito de discutir las implicaciones del retorno y la reinserción social y laboral en el actual escenario de la crisis económica de los mercados y la implementación de políticas de reforzamiento de la seguridad en la frontera México-Estados Unidos. El objetivo principal es analizar el retorno como resultado de la articulación y dinámica de mercados de trabajo globales.

Palabras clave: migración de retorno, reinserción social, reinserción laboral, zona metropolitana, México

\begin{abstract}
This article examines the process and contemporary conditions of the return of Mexican migrants from the United States in urban contexts, in order to discuss the implications of the return and social and labor reintegration in the current scenario of the economic crisis of the markets and implementation of policies reinforcing security in the Mexico-US border. The main objective is to analyze the return as a result of the articulation and $d y$ namics of global labor markets.
\end{abstract}

Key words: return migration, social reintegration, labor reintegration, metropolitan area, Mexico

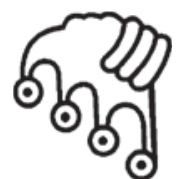

IZTAPALAPA

Agua sobre lajas

* Los hallazgos de investigación que se presentan en este artículo son resultado del proyecto Conacyt núm. 105357, Movilidades y establecimiento. Migrantes retornados en Nezahualcóyotl, ¿Quemar las naves o re-emigrar?, Consejo Nacional de Ciencia y Tecnología, 2010-2014.

${ }^{* *}$ Profesora-investigadora del Centro de Estudios Sociológicos, El Colegio de México rivesanl@colmex.mx 


\section{Introducción}

e presentan resultados de una investigación en curso relativa a las expe- riencias de retorno y a la reinserción social y laboral de los migrantes que vuelven de Estados Unidos a la Zona Metropolitana de la Ciudad de México. El artículo se organiza en cinco apartados. El primero presenta de manera breve algunos supuestos que sustentan la investigación acerca de las modalidades contemporáneas de reinserción, establecimiento y reemigración de los migrantes retornados a Nezahualcóyotl, Estado de México. En esta sección se establecen las bases para examinar las experiencias de los retornados en un contexto urbano-metropolitano desde la perspectiva global de las migraciones. El segundo apartado ofrece algunos antecedentes de la formación histórica del municipio de Nezahualcóyotl y su dinámica territorial; y, en el tercero, se presenta un análisis de las características sociodemográficas de los habitantes del municipio, que evidencian una dinámica de alta movilidad poblacional. A partir de la conformación de la población local es posible entender cómo se interrelacionan la migración interna con la internacional y el retorno en esa localidad, así como las movilidades intraurbanas provocadas por la proximidad con la Ciudad de México. Este contexto de retorno hace posible establecer, en el cuarto apartado, los rasgos de empleo, ocupación y escolaridad de la población inmigrante interna y retornada internacional a esa localidad. Finalmente, en el quinto $y$ último apartado se presentan y analizan experiencias de retorno y reinserción social y laboral en el municipio, para lo cual se construyen dos casos prototípicos de retornados internacionales recientes (llegados en los últimos cuatro años) con el objetivo de estudiar las posibles rutas y resultados de esa experiencia. Es importante señalar que este documento constituye una aproximación a estos sujetos de estudio y no pretende establecer generalizaciones. 


\section{Los puntos de partida y las coordenadas metodológicas}

En este apartado se presentan esquemáticamente algunas consideraciones que sustentan esta investigación y dan cuenta de las modalidades que adquieren los procesos de inserción social y laboral de los migrantes de retorno internacional en un contexto urbano. El trabajo se desarrolló en la Zona Metropolitana de la Ciudad de México, en particular en Nezahualcóyotl, un municipio conurbado del Estado de México localizado al oriente del Distrito Federal.

La escasa producción académica sobre las experiencias y los efectos de los retornados en centros urbanos de México, sobre todo en las áreas metropolitanas, donde hoy en día -según datos del Instituto Nacional de Estadística y Geografía (INEGI, 2000, 2005 y 2010)- se perfilan algunas concentraciones significativas de inmigrantes retornados recientes, motivó que, en enero de 2008, diseñara una propuesta de investigación sobre el retorno y la reinserción. Hasta ese momento, la mayoría de los estudios sobre los retornados en México se habían realizado en localidades rurales y sobre todo en el occidente del país, región que coincide con la llamada zona tradicional e histórica de la migración mexicana a Estados Unidos (Espinosa, 1998; Canales, 1999; Papail, 2002 y 2005; Durand y Massey, 2003). En la actualidad, después de la crisis de los mercados de trabajo internacional y de sus efectos visibles en la dinámica de los flujos migratorios y en los mercados, y del endurecimiento de las leyes migratorias en Estados Unidos, se advierte la necesidad de observar otras regiones no sólo en México sino en América Latina, $y$ las investigaciones sobre el retorno contemporáneo de inmigrantes ya han tomado nuevos derroteros; ${ }^{1}$ asimismo, se evidencia un mayor interés de los académicos sobre las migraciones y las movilidades humanas así como en el retorno en contextos urbanos (cf. Aznar, 2011; Ibarra Templos, 2011; Herrera et al., 2013; Villa, 2013).

Este vacío en el campo de las migraciones me impulsó a realizar un estudio comparativo de los efectos y las características de los migrantes retornados en diferentes periodos de salida y de retorno. Es decir, aquellos del Programa Bracero (1942-1964) y los contemporáneos -quienes empezaron a emigrar de Nezahualcóyotl, por ejemplo, a principios de la década de los ochenta (cf. Rivera Sánchez, 2008)-. Ese objetivo, ambicioso para un periodo de tres años, ${ }^{2}$

1 Véase por ejemplo los diversos artículos que integran el volumen de julio-diciembre de 2013 de la Revista Interdisciplinar da Mobilidade Humana, cuyo dossier es sobre "Retorno e Circularidade".

2 Duración de un proyecto de investigación en ciencias básicas con financiamiento del Consejo Nacional de Ciencia y Tecnología (Conacyt). 
condujo a introducir una perspectiva histórica de largo plazo en el diseño de la investigación, tanto de las emigraciones como del retorno, para detectar continuidades y cambios entre cada periodo, a partir de las trayectorias laborales y migratorias de los retornados internacionales y de los censos 2000, 2005 y 2010, que contienen información relativa a los retornados y sus hogares de inserción.

La reflexión sobre las temporalidades de las vivencias migratorias y laborales condujo además a incluir ciertas dimensiones espaciales relevantes para el debate sobre la inserción de los inmigrantes en este municipio mexiquense, priorizando en esencia una exploración sobre el rol que podría desempeñar el lugar de origen $y / o$ salida hacia un destino internacional en la conformación de la experiencia migratoria y laboral, y en particular en las modalidades de inserción social y laboral al retorno. Es decir, quienes salieron de este municipio, así como quienes emigraron de otro sitio, pero que volvieron a este espacio urbano. Un estudio de este tipo permitiría comparar las trayectorias de los retornados que han salido de Nezahualcóyotl y de quienes lo han hecho desde otras ciudades del país, así como de quienes han emigrado de localidades rurales y que, después de vivir en Estados Unidos, se establecen en el municipio de Nezahualcóyotl.

Un principio nodal que inspiró esta investigación fue tomar distancia metodológica de aquellas que consideran como unidades de análisis y de referencia para estudiar el proceso migratorio sólo un lugar de origen y un lugar de destino - una modalidad típica de las investigaciones sobre el tema- y que, en consecuencia, asumen que el retorno ocurre necesariamente al lugar de origen. Esta estrategia conduce a observar sólo a personas procedentes del mismo sitio, estudiarlas como "un grupo étnico", un grupo o un agregado de personas, volviendo esencial el lugar de procedencia en cuanto marcador de homogeneización social y cultural que, si bien es relevante por su efecto en la constitución de redes sociales y vínculos, no es crucial. Lo anterior podría llevar a suponer a priori que los migrantes, en este caso los retornados, comparten trayectorias y prácticas culturales asociadas a la localidad desde la cual se desplazaron, y aunque esto podría implicar compartir ciertos códigos socioculturales, reflejados en la formación de redes de proximidad y ciertos valores y tradiciones regionales que han influido en su socialización, no por fuerza tienen el mismo resultado en términos de las trayectorias vitales y los mecanismos de inserción social y laboral desarrollados.

Es decir, en términos metodológicos se pretende un alejamiento de las unidades de análisis entendidas como "contenedores" de relaciones sociales y se adopta a la ciudad inmersa en la zona metropolitana y conectada a diversos lugares de procedencia de los migrantes internos $y$ de retornados de varios lugares de 
destino en Estados Unidos, a fin de verificar en este sitio (contexto de retorno) la complejidad y la diversidad del fenómeno en estudio (cf. Glick Schiller y Caglar, 2007; Glick Schiller, 2007).

Se busca profundizar en el conocimiento de los retornados no como "un grupo" o agregado, con atributos o formas de reinserción homogéneas o similares, sino como individuos de distintos lugares de origen, inmersos en redes y campos sociales diversos, y que comparten la experiencia de regresar de Estados Unidos a Nezahualcóyotl, pero cuyas motivaciones para volver, su trayectoria migratoria y experiencia de retorno, así como sus procesos de reinserción laboral y social, difieren en cuanto a las consecuencias individuales/familiares y a su posible impacto social. En suma, los efectos del retorno también pueden ser variados, teniendo en cuenta el capital social, la posición en los campos sociales y la relación con los contextos y las condiciones que median su retorno (si se trata de devueltos, repatriados, deportados o "voluntarios"), entre otros factores de diferenciación social.

En este estudio no se busca clasificar a los retornados en alguna de las dos categorías sociales con las que comúnmente se han etiquetado:"exitosos" $y$ "fracasados" (cf. Cassarino, 2004; Durand, 2004). La propia escala de la ciudad-como sitio de prueba para la investigación-, contenida en la zona metropolitana ${ }^{3} y$ atravesada por movilidades diversas, ofrecerá elementos para problematizar los resultados y la experiencia de retorno internacional.

En síntesis, se propone entender el contexto de retorno y el papel de los retornados como agentes sociales que median entre las condiciones estructurales y las subjetivas/objetivas que se entretejen en la experiencia para diseñar estrategias de reinserción.

Por lo tanto, se analiza el retorno como parte del proceso migratorio y no en cuanto conclusión definitiva del proyecto como migrante; esto es, el retorno no necesariamente es definitivo y permanente, sino una fase del proceso migratorio, que tiene efectos (igual que la emigración) sobre las personas y los lugares, y por ello debe estudiarse de manera longitudinal (Rivera Sánchez, 2011).

El estudio cuyos resultados se presentan toma al municipio de Nezahualcóyotl como un lugar de retorno que puede ser asimismo, en el itinerario de los migrantes, el lugar de origen, de salida (para inmigrantes que previamente migraron

3 Para una explicación sobre las escalas y el papel de las ciudades con presencia de población inmigrante en los procesos de incorporación o inserción laboral y social, véase la introducción, además de los capítulos 4 y 10 de Glick Schiller y Caglar (2011). 
en el país, además de al extranjero), de paso, o uno para realizar una escala temporal antes de regresar a la localidad de origen, reemigrar a un destino interno o hacerlo nuevamente a uno internacional (cf. Rivera Sánchez, 2008).

Una apuesta de esta investigación es que un análisis con las peculiaridades señaladas contribuye a entender los procesos de interconexión entre la migración interna y la internacional en México, y a explicar su concatenación histórica. Sin duda, el municipio de Nezahualcóyotl ha sido un lugar de destino relevante para los migrantes procedentes de distintos estados y municipios del país, así como del Distrito Federal; baste señalar que en Nezahualcóyotl hay pobladores de todos los estados del país (cf. Rivera Sánchez, 2008). Además, el número de migrantes que salen de Nezahualcóyotl a Estados Unidos había tenido un crecimiento constante hasta el año 2000, y se había observado un incremento sostenido de la población que regresaba de Estados Unidos a Nezahualcóyotl. En los siguientes acápites se mostrará cómo se han modificado estas tendencias.

Finalmente, un estudio sobre la reinserción social y laboral de los migrantes retornados a un espacio urbano permitirá observar los efectos sobre la probabilidad de conseguir empleo en el mercado de trabajo local; las formas en que funciona el capital social, la escolaridad $y$, en general, las habilidades y destrezas adquiridas en la estancia como inmigrantes internacionales, una vez que regresan a México. Es decir, permite poner a prueba la tesis sobre el capital humano acumulado para observar su papel en el proceso de reinserción laboral. Asimismo, un análisis que tenga en cuenta el tipo de hogar de inserción y sus dinámicas puede ofrecer evidencia de las relaciones familiares y personales del retornado, el proceso de reajuste a otra dinámica social en un contexto (ahora de retorno) que sin duda ha cambiado, no obstante que el migrante hubiera salido de ese mismo sitio. De igual modo, es posible explorar el efecto de la readaptación o la reincorporación, del ajuste de expectativas o de conflicto que generan las movilidades humanas.

Una investigación como ésta puede contribuir, por lo demás, a comprender el efecto del retorno de los migrantes internacionales en un contexto urbano, localizado en la Zona Metropolitana de la Ciudad de México. Esto es, someter a comprobación la hipótesis de que, en México, el efecto de la migración internacional ( $y$ en particular de los retornados como agentes sociales) "se diluye" en el contexto urbano $y$, sobre todo, en un contexto metropolitano de alta densidad poblacional, y que sólo se refleja con nitidez en las localidades rurales alejadas de las zonas metropolitanas, mediante cambios evidentes en los paisajes locales y la dinámica comunitaria. 


\section{El municipio de Nezahualcóyotl, Estado de México: dinámica poblacional}

Nezahualcóyotl fue fundado el 3 de abril de 1963. Los terrenos que hoy lo conforman pertenecen históricamente al Lago de Texcoco en la Zona Metropolitana de la Ciudad de México, lago que fue desecado entre 1912 y 1932. Una vez concluido el proceso de desecación iniciaron los asentamientos en esa región. Los terrenos de propiedad federal empezaron a venderse a bajo costo, pues carecían de servicios públicos básicos. Así, en la década de los cuarenta, con la construcción del bordo de Xochiaca y el túnel de Tequixquiac, se generó la afluencia masiva de habitantes de la Ciudad de México, de otros municipios metropolitanos y de otras entidades del país, que buscaban asentarse en la zona metropolitana y que contaban con pocos recursos para hacerlo. Con este proceso inició la formación de las primeras colonias, entre las que se encuentran Juárez Pantitlán, México, El Sol, El Barrio de Juárez o San Juan, por mencionar algunas de las tradicionales. En 1953 ya existían 13 colonias proletarias sumamente pobladas. Seis años más tarde, en 1959, había 33 colonias. La explosión demográfica y la falta de servicios e infraestructura urbana generaron una gran organización de los pobladores, quienes comenzaron a demandar que las colonias se organizaran en fraccionamientos habitacionales para generar una estructura de representación en la gestión urbana del territorio, y exigir, a través de esta estructura, la atención gubernamental, no sólo federal, sino del Estado de México (Secretaría de Gobernación, 1999)

La reorganización de las colonias en el antiguo vaso de Texcoco provocó cierto proceso de reordenamiento territorial y, en 1963, uno de los cuatro principales fraccionamientos de colonias de la región Texcoco fue separado administrativamente del municipio de Chimalhuacán, y se constituyó entonces como un municipio independiente con el nombre de Nezahualcóyotl. No obstante, el proceso de lotificación y regularización de los terrenos ha sido largo, y hasta la década de los noventa la regularización de la tenencia de la tierra, el uso del suelo y la dotación de servicios públicos fue una demanda constante de sus habitantes.

En la actualidad, el municipio es parte de la zona conurbada de la Ciudad de México, en la porción oriental del Valle de México. Está compuesto por dos localidades, la más importante es la cabecera municipal, con el mismo nombre (conocida como Ciudad Nezahualcóyotl), que concentra a la mayoría de la población (99.9\%) y que se distribuye a lo largo de 86 colonias urbanas. El total de la población en 2010 fue de 1107931 habitantes, según el Censo de Población y Vivienda (INEGI, 2010). Por su ubicación en la zona metropolitana y el hecho 
de ser un municipio de relativa reciente creación, los habitantes provienen de casi todos los estados del país; es decir, el municipio es producto, por un lado, de la migración interna (del campo a la ciudad) hacia la Zona Metropolitana del Valle de México y, por otro, de cierto proceso de precarización de los habitantes de la capital y otros municipios metropolitanos del Estado de México que, entre 1970 y 1980, vieron mermadas sus condiciones de vida y se desplazaron hacia la periferia (Lindón, 1999). Entre 1970 y 1980, la tasa de crecimiento poblacional fue de $8.7 \%$, prácticamente el doble del promedio estatal. Pero a partir de los ochenta el decrecimiento ha sido constante.

\section{El contexto de retorno: notas sociodemográficas}

Hoy en día, los procesos de migración interna en México constituyen un tema poco estudiado, si se compara con el volumen alcanzado durante la última década por los relativos a la migración internacional México-Estados Unidos y por aquellos que tratan la migración interna, realizados en las décadas de los setenta y los ochenta, periodo asociado a los procesos de urbanización y formación de las zonas metropolitanas en México y en otros países de América Latina (Rivera Sánchez, 2012b). La migración interna en cuanto proceso y la presencia de migrantes procedentes de otras regiones de México en los municipios metropolitanos y particularmente en el de Nezahualcóyotl resultan vitales para esta investigación, debido a que el origen estatal de los radicados en este municipio permite, en determinados casos, entender ciertas lógicas que mueven a emigrar a Estados Unidos, trazando o dándole seguimiento a rutas y destinos migratorios recurrentes entre la población municipal, a la vez que posibilita observar cómo esta estructura de redes sociales influye en las motivaciones de retorno (cuando éste es voluntario), por ejemplo en la elección del lugar para volver al país. La dinámica de la migración interna se ha entrelazado con la internacional y el retorno a México, sin que ello signifique que el lugar de procedencia es un marcador de homogeneización sociocultural que determina los cursos de acción de los migrantes (Rivera Sánchez y Lozano Ascencio, 2006).

Nezahualcóyotl, al igual que otros municipios metropolitanos del Estado de México, ha sido lugar de destino recurrente para los migrantes procedentes de distintos estados y municipios del país, así como del Distrito Federal. Además, hasta el año 2000 fue expulsor constante de su población hacia Estados Unidos. Lo mismo había ocurrido con la población que retorna de ese país a este 
municipio. No obstante, es relevante anotar que la población total de Nezahualcóyotl ha descendido en las últimas tres décadas, ${ }^{4}$ como consecuencia de un ensanchamiento del área metropolitana y de un proceso de relocalización de la población en la zona conurbada con el Distrito Federal, lo que ha implicado desplazamientos poblacionales hacia municipios aledaños como Chimalhuacán, Ixtapaluca, Los Reyes y Chalco Solidaridad, en el Estado de México. Un dato interesante de la población que residía en 2010 en Nezahualcóyotl es que 60\% del total no había nacido en la entidad donde se localiza. El primer grupo de personas no nacidas en el Estado de México estaba integrado por originarios del Distrito Federal, que en 2010 representaron 40\% del total de la población, un porcentaje similar al de los nacidos en el Estado de México; el resto se distribuye entre quienes nacieron en otras entidades del país. En orden de importancia se encuentran los nativos del estado de Puebla, seguidos por los de Veracruz, Oaxaca y Michoacán (por mencionar los principales), todos ellos con una representación porcentual menor a 5\% respecto del total de habitantes (INEGI, 2010).

Así, Nezahualcóyotl experimenta una intensa movilidad poblacional, en diversas modalidades, direcciones y de forma simultánea: emigración hacia otros municipios de la zona metropolitana y hacia el Distrito Federal, además de otros destinos internacionales (a Estados Unidos principalmente), y sigue manteniendo una dinámica de inmigración constante de nuevos pobladores procedentes del interior del país, ${ }^{5}$ además de ser receptor de personass de retorno internacional.

En relación con quienes viajan a Estados Unidos saliendo de Nezahualcóyotl en la última década, es notable el descenso que registró el Censo de Población entre 2000 y 2010. Entre 2005 y 2010 salieron menos personas con destino a Estados Unidos (63\% menos) que quienes lo hicieron entre 1995 y 2000. Por el contrario, entre los migrantes de retorno del quinquenio 1995-2000 y los del periodo 2005-2010 se observa un gran incremento, los retornados en este último periodo representaron $40 \%$ más. Pero sin duda el incremento más significativo es el registrado al comparar la cantidad de retornados de 2000 a 2005 respecto de quienes volvieron en el lustro siguiente (2005-2010), la diferencia es de $66 \%$ más en este último (INEGI, 2010), lo cual muestra que el retorno migratorio

4 En 2010 Nezahualcóyotl registró una población total de 1107931 habitantes, y en 2000 había registrado 1225972 habitantes, es decir, tuvo un decremento de nueve puntos porcentuales en una década (INEGI, 2000 y 2010).

5 Destacan los inmigrantes llegados a Nezahualcóyotl en los últimos cinco años procedentes de los municipios metropolitanos, como Ecatepec de Morelos, Chimalhuacán, Ixtapaluca y Chalco, del Estado de México, así como de las delegaciones Iztapalapa, Gustavo A. Madero y Venustiano Carranza, del Distrito Federal (INEGI, 2010). 
a Nezahualcóyotl ha aumentado durante la última década y con mayor énfasis entre 2005-2010, periodo que coincide con la agudización de la crisis económica en Estados Unidos y la evidente contracción de los mercados de trabajo, sobre todo de los no especializados, donde se insertan los indocumentados. ${ }^{6}$

Asimismo, este mayor volumen de retornados en el último quinquenio respecto de los dos previos podría reflejar el efecto de una política migratoria restrictiva en algunos estados fronterizos de Estados Unidos (como parte del programa denominado Comunidades Seguras), políticas que han generado condiciones adversas para los inmigrantes indocumentados, sin importar los años de estadía y la experiencia como inmigrante (redadas en espacios laborales, deportaciones y repatriaciones, disposiciones legales que restringen algunos derechos sociales, entre otras), el reforzamiento de la frontera entre México y Estados Unidos, así como la saturación de las fuentes de trabajo para mano de obra no calificada en los países centrales (sobre todo en los sectores de los servicios y la manufactura), como lo han señalado algunos especialistas a propósito de la dinámica de los mercados de trabajo estadounidenses desde 2008 (Durand, 2009).

Si comparamos el perfil de los retornados del periodo 1995-2000 con los de 2005-2010 destaca lo siguiente: las personas retornadas de Estados Unidos a Nezahualcóyotl, según datos del año 2000, se concentraban en los segmentos poblacionales que incluyen, además de los niños de entre cinco y nueve años, a los adultos de entre 20 y 39 años de edad, y de manera notable el segmento de entre 25 y 29 años; es decir, los retornados se encuentran en plena edad productiva y aún en el rango de edad considerado como joven (menores de 30 años). Se trataba en su mayoría de hombres, aunque también se detectó un número significativo de mujeres de entre 20 y 34 años. En suma, quienes volvieron a Nezahualcóyotl registrados en 2000 eran $62 \%$ hombres y $38 \%$ mujeres, y tenían en promedio 30 años de edad. En 2010, la estructura por sexo y edad de la población retornada al municipio se distribuyó en $65 \%$ hombres y $35 \%$ mujeres, disminuyendo ligeramente la participación de las mujeres respecto de los datos de 2000 (INEGI, 2000 y 2010). El promedio de edad se mantuvo en 30 años (mediana de edad), y las mujeres son más jóvenes (cuatro años en promedio) que los hombres retornados.

Un cambio en la estructura de la población retornada a Nezahualcóyotl según las cifras de 2010 en relación con las de 2000 se observa en los rangos etarios

6 Según el INEGi (2012), el saldo neto migratorio internacional en México, al primer trimestre de 2012, es prácticamente nulo. De acuerdo con el análisis de la serie histórica, durante 2010, en México se perdió menos población por efecto de la migración internacional ( 8.5 por cada diez mil) y, aunque se recuperó para 2011 (11.6), la tasa de emigración a Estados Unidos todavía se encuentra por debajo de la tasa promedio registrada para 2009, que fue de 13.9 (cf. INEGI, 2012). 
en los que se concentra: entre los 25 y 39 años de edad en 2010; mientras que en 2000 se mantenía una fuerte participación entre los segmentos de 20 y 25 años. En 2010 se concentran en el rango inmediato superior, pero sobre todo se distribuyen en dos segmentos de edad, el de 25 a 29 años y el de 35 a 39 años, tanto para hombres como para mujeres. En síntesis, se puede afirmar que no ha variado significativamente el perfil de edad y sexo de la población retornada a Nezahualcóyotl en los últimos 15 años; continúan agrupándose en los rangos de edad productiva, incluyendo uno de los segmentos que caracteriza a la población considerada como joven (25-29 años), no obstante que se observa una tendencia a concentrarse en los rangos de edad subsecuentes (mayores) en 2010. Además, los segmentos en los que predominan las mujeres retornadas son los que comprenden entre los 40 y 44 años y el de entre 10 y 14 años (INEGI, 2010).

En la población retornada entre 1995 y 2000, en cuanto a la relación de parentesco que guarda con el(la) jefe(a) del hogar, se observa que más de la tercera parte de esta población son hijos(as) (36.2\%), 32\% son jefes del hogar que al retornar asumen esa posición, $18.6 \%$ tiene otro vínculo de parentesco y $12 \%$ son esposo(a) o pareja del (de la) jefe(a) del hogar. En cuanto al estado conyugal de los retornados, la mayoría de ellos son personas unidas (58.9\%). Si se compara la posición que guardan los retornados captados en 2000 con los del Censo de Población y Vivienda de 2010, se encuentra que $45 \%$ de la población retornada en el lustro 2005-2010 son hijos(as) del (de la) jefe(a) del hogar y 33\% son efectivamente jefes(as) del hogar, $13 \%$ son yernos/nueras del (de la) jefe(a) del hogar en el que vivían en 2010, y 9\% tiene otro parentesco. En este aspecto se revela un notable cambio en la relación del retornado con el jefe del hogar en el que vivía cuando se levantó el registro censal en 2010. Es decir, ha aumentado la posición de hijo entre quienes han retornado en el lustro 2005-2010 respecto de los dos precedentes.

Esta observación sobre el lugar del retornado en el hogar no es ociosa, en alguna forma podría ayudarnos a entender la lógica de la reemigración, pues se trata de personas que aún no han formado su propio hogar, que se encuentran unidos y están conformando una familia nuclear, pero que están dentro de otro hogar, o que no cuentan con medios suficientes para habitar en otra vivienda.

Además, cabe señalar que $39 \%$ de las personas que regresaron de Estados Unidos a Nezahualcóyotl durante el periodo 2005-2010 son originarios del Estado de México, y 38\% del Distrito Federal; es decir, 77\% es originario de municipios metropolitanos y $23 \%$ proviene de otras entidades, entre las que destacan en orden descendente Michoacán, Guerrero, Oaxaca, Puebla y Yucatán (cuadro 1). 
Cuadro 1

Inmigrantes de retorno de Estados Unidos en Nezabualcóyotl, Estado de México, según entidad de nacimiento, 2000 y 2010

(distribución porcentual)

\begin{tabular}{lcc}
\hline Entidad federativa & $\begin{array}{c}1995-2000 \\
(\%)\end{array}$ & $\begin{array}{c}2005-2010 \\
(\%)\end{array}$ \\
\hline Estado de México & 31.6 & 38.9 \\
Distrito Federal & 41.9 & 37.8 \\
Michoacán & 4.6 & 6.1 \\
Guerrero & 2.9 & 3.2 \\
Oaxaca & 3.8 & 2.9 \\
Puebla & 5.5 & 2.8 \\
Guanajuato & 3.0 & $*$ \\
Otros estados & 6.8 & 8.4 \\
\hline Total & 100 & 100 \\
\hline
\end{tabular}

* Es menor a 2\%, por lo que no es significativo. Se han incluido porcentajes mayores a $2 \%$. Fuente: Elaboración propia con base en INEGI (2000 y 2010).

Estas proporciones muestran que al menos $61 \%$ de la población retornada a Nezahualcóyotl tiene también experiencia migratoria interna, además de la internacional. Lo anterior significa que seis de cada 10 retornados han experimentado al menos dos viajes, uno nacional, al mudarse de otra entidad a Nezahualcóyotl, y por lo menos uno internacional, cuando emigraron a Estados Unidos. Esta experiencia migratoria podría ser también relevante en la constitución de cierto capital para conseguir un empleo. A continuación intentaremos dar cuenta de si este factor desempeña una función en ese proceso de reinserción laboral.

\section{Empleo y escolaridad entre la población inmigrante reciente en Nezahualcóyotl: retornados e inmigrantes internos}

Hemos considerado la escolaridad de la población de retorno a partir del registro censal, teniendo en cuenta el número de años estudiados y aprobados en el sistema escolar, por lo que sólo se incluye la población de 15 años y más. En el 2000 el promedio de escolaridad de los retornados a Nezahualcóyotl fue de 7.6 años de estudio, para 2010 el promedio alcanzó 9.2 años (mediana), y se mantuvo similar la distribución entre hombres y mujeres, con una ligera diferencia no 
significativa (a la baja) en la escolaridad en el caso de las mujeres (INEGI, 2000 y 2010). Este promedio de años de estudio implica que los retornados registrados en 2010 han cursado la secundaria como máximo nivel de estudio, recordemos que la edad promedio de esta población es de 30 años, y que en su mayoría se posicionan como hijo(a) en el hogar en el que vivía en 2010.

Por otra parte, para complementar el perfil del retornado se exploraron los datos censales relativos a la condición laboral de las personas que cinco años atrás vivían en Estados Unidos y que en 2010 habían regresado a Nezahualcóyotl. ${ }^{7}$ Es oportuno anotar que, en este caso, la referencia de su actividad laboral se refiere a su experiencia de trabajo desde su arribo a Nezahualcóyotl, es decir que el tiempo máximo que se registra en la actividad laboral es de cinco años (INEGI, 2010). Para ello, se examinan dos variables censales que permiten dar cuenta de si la población de retorno ha trabajado desde que regresó de Estados Unidos y en qué tipo de empleo se ha ocupado (posición en el empleo). Así, del total de los retornados en 2005-2010, ${ }^{8} 70 \%$ ha trabajado desde su llegada al municipio, mientras que para el periodo 1995-2000, 73\% lo había hecho. No obstante, no todos quienes declararon haber trabajado en ese periodo de cinco años anteriores a 2010 realizaron actividades económicas, sólo 56\% dijo haber trabajado en actividades económicas remuneradas (en este rubro 7.3 de cada 10 eran hombres y el resto mujeres), 15\% aproximadamente se ocupó realizando quehaceres del hogar - una actividad no remunerada económicamente-, mientras que $29 \%$ aseguró que no trabajó desde su arribo a Nezahualcóyotl (cuadro 2).

\section{CuAdro 2}

Inmigrantes de retorno de Estados Unidos en Nezahualcóyotl, Estado de México, según condición de actividad, 2000 y 2010

(distribución porcentual)

\begin{tabular}{lcc}
\hline Tipo de actividad & $\begin{array}{c}1995-2000 \\
(\%)\end{array}$ & $\begin{array}{c}2005-2010 \\
(\%)\end{array}$ \\
\hline Actividades económicas remuneradas & 53.6 & 56.0 \\
Actividades no remuneradas & & \\
(en negocio o predio familiar) & 18.4 & 15.0 \\
No trabajó & 28.0 & 29.0 \\
\hline Total & 100 & 100 \\
\hline
\end{tabular}

Fuente: Elaboración propia con base en INEGI (2000 y 2010).

Ésta es la definición de retornado que tiene en cuenta el Censo de Población en cada quinquenio.

En este caso se considera exclusivamente a la población retornada de 12 años y más. 
Del total de mujeres que regresaron a Nezahualcóyotl en este último quinquenio, más de $44 \%$ se concentró en quehaceres del hogar, 33\% no trabajó y sólo $20 \%$ realizó actividades económicas remuneradas.

Estas variables censales son significativas porque ponen de manifiesto no sólo si los retornados han desarrollado actividades económicas desde su regreso a Nezahualcóyotl, sino también en qué posición en el empleo se insertaron cuando realizaron actividades económicas remuneradas ${ }^{9}$ y cómo se distribuyen entre hombres y mujeres. Del total de quienes volvieron de Estados Unidos entre 2005 y 2010 y que laboraron en actividades remuneradas, tenemos que la mitad se posicionó como empleado u obrero y $39 \%$ como "trabajador por cuenta propia". Entre los primeros, $90 \%$ eran hombres y sólo $10 \%$ mujeres. Mientras que de los trabajadores por cuenta propia, $81.5 \%$ eran varones y $18.5 \%$ mujeres. De las mujeres retornadas que se emplearon en actividades remuneradas, $59 \%$ lo hizo como trabajadora por cuenta propia y $40 \%$ como empleada u obrera.

Por último, en cuanto a las posiciones en el empleo nominadas como "jornalero y/o peón", por un lado, y "patrón y empleador", por el otro, los porcentajes no son significativos, sobre todo en la segunda donde la cifra fue prácticamente nula en los registros censales de 2010.

Al comparar la población retornada en 2005-2010 con los inmigrantes internos que llegaron en ese mismo quinquenio a Nezahualcóyotl, se encuentra que el número de años de escolaridad promedio es similar. Asimismo, entre los inmigrantes internos recientes sólo $18 \%$ afirmó no haber trabajado en esos cinco años, frente a $29 \%$ que declaró no haberlo hecho en ese periodo. A la vez, $82 \%$ de los inmigrantes internos en Nezahualcóyotl afirmó haber trabajado (frente a 70\% en el caso de los retornados) y, de éstos, 63\% laboró en actividades económicas remuneradas (frente a 56\% de los retornados), $72 \%$ se desempeñó como empleado u obrero y alrededor de $20 \%$ fue "trabajador por cuenta propia" (los porcentajes para los retornados son 50 y $39 \%$, respectivamente).

Esta mirada comparativa demuestra que los inmigrantes procedentes de otros estados y municipios del país trabajaron en mayor proporción que los retornados de Estados Unidos en ese periodo, no obstante que ambos inmigrantes (internos e internacionales) arribaron a Nezahualcóyotl en el mismo lustro (2005-2010). Se realiza el parangón entre estas dos poblaciones precisamente porque ambas comparten la peculiaridad de haber llegado a Nezahualcóyotl y explorado el mercado de trabajo en los mismos años. En el cuadro 3 se resume la comparación porcentual entre las dos categorías principales de empleo y ocupación en 2010.

9 Recuérdese que "quehaceres del hogar" es considerada una actividad no económica por no ser remunerada. 


\section{CuAdro 3}

Inmigrantes internos y retornados de Estados Unidos en Nezahualcóyotl, Estado de México, según posición en el empleo, 2010

(distribución porcentual de las principales categorías censales)*

\begin{tabular}{lcc}
\hline $\begin{array}{c}\text { Posición en el empleo } \\
2005-2010\end{array}$ & $\begin{array}{c}\text { Inmigrantes } \\
\text { internos }\end{array}$ & $\begin{array}{c}\text { Retornados de } \\
\text { Estados Unidos }\end{array}$ \\
\hline & $\begin{array}{c}\text { (63\% ha realizado actividades } \\
\text { económicas remuneradas })\end{array}$ & $\begin{array}{c}\text { (56\% ha realizado actividades } \\
\text { económicas remuneradas })\end{array}$ \\
Empleado u obrero & 72 & 50 \\
Trabajador por cuenta propia & 20 & 39 \\
\hline
\end{tabular}

* No se incluyen en el cuadro categorías como patrón, jornalero o peón y no especificados; en ninguno de esos casos alcanza 3\%.

Fuente: Elaboración propia con base en INEGI (2000 y 2010).

Las explicaciones de esta diferencia pueden ser diversas, a continuación se sugieren algunas hipótesis: en ciertos casos, los retornados trajeron consigo ahorros de Estados Unidos y ello les permitió no trabajar durante varios meses, o bien enfrentaron mayores dificultades para conseguir un empleo en el mercado de trabajo una vez que llegaron de Estados Unidos, frente a quienes arribaron procedentes del interior del país. Otra hipótesis combina las dos anteriores: los retornados trajeron consigo recursos, enfrentaron dificultades en el mercado de trabajo y decidieron emplearse por cuenta propia, quizá usando los recursos económicos obtenidos durante su experiencia como inmigrantes en el extranjero o poniendo a prueba las habilidades y destrezas adquiridas.

Adicionalmente, es notable la marcada diferencia entre los retornados que se concentran en las categorías de "empleado u obrero" y "trabajador por cuenta propia" y los inmigrantes internos en Nezahualcóyotl que ocuparon esas posiciones en el empleo. Esta última posición incluye a carpinteros, plomeros, herreros, comerciantes, vendedores por catálogo o ambulantes, propietarios de un taxi que ellos mismos conducen, tenderos, taqueros, entre otros muchos oficios $y$ actividades de autoempleo; se trata de personas que desempeñan algún oficio o tienen algún negocio -sin empleados- de donde obtienen su ingreso cotidiano, el cual es variable. En esta categoría tenemos un gran volumen de inmigrantes de retorno internacional en Nezahualcóyotl entre 2005-2010.

Con base en los datos expuestos es posible comparar las características sociodemográficas de retornados e inmigrantes internos en los tres últimos 
quinquenios censales, pero no puede realizarse un análisis longitudinal de su comportamiento demográfico, por lo que se propuso como una parte medular de esta investigación complementar el estudio examinando trayectorias migratorias y laborales de los retornados, utilizando un instrumento diseñado ex profeso para construir trayectorias vitales (cuyo recorte metodológico está dado por el inicio de la experiencia laboral y la realización del primer viaje desde su lugar de origen) a partir de entrevistas de corte biográfico. Pero debido a que esta labor no ha concluido, en este artículo se revisan trayectorias de los retornados a Nezahualcóyotl mediante la construcción de dos casos prototípicos, los cuales representan dos modalidades tipológicas de máxima diferencia en cuanto al resultado de la inserción laboral. El primer caso es el del retornado que reemigra a Estados Unidos, y el segundo el del retornado que quema las naves para insertarse laboralmente.

Antes de presentar los casos, es pertinente señalar que las entrevistas a retornados contemporáneos fueron realizadas en las diferentes colonias del municipio, y esto responde al hecho de que en las colonias de Nezahualcóyotl existen grandes concentraciones de personas procedentes de varios estados del país, y su localización podría dar cuenta de ciertas redes de migrantes y de retornados que comparten el mismo origen estatal (esto se constató, además, con los datos censales de las últimas tres décadas). Así, con el fin de no concentrar las entrevistas en una misma red de migrantes a partir de la técnica de bola de nieve, se decidió georreferenciar los datos censales de los inmigrantes internos y de los retornados internacionales en tres momentos, los años 2000, 2005 y 2010 , $y$, en efecto, se encontró que inmigrantes procedentes de un mismo estado se concentraban -de manera notable- en ciertas demarcaciones o colonias (áreas geoestadísticas básicas -AGEB-), lo que no excluía la presencia de inmigrantes de otros estados y municipios. Con base en esa información, se efectuó un muestreo teórico que condujo a considerar ciertos perfiles de migrantes de retorno, en los que, además de su referencia al lugar de origen, se incluye su localización espacial en el municipio, lo que ha hecho más compleja la investigación en campo. Los relatos biográficos de inmigrantes de retorno reciente en Nezahualcóyotl, que se incluyen en el siguiente apartado, tienen como propósito abundar en las condiciones y los factores que intervienen en el proceso de reinserción laboral (y también social). Por supuesto, no se trata de estandarizar la experiencia humana, la cual es particular $y$ única en cada caso, sino de reflexionar, a partir de estos dos casos prototípicos, sobre esos factores condicionantes estructurales y subjetivos del retorno y la reinserción, y lo que de ahí resulte. 


\section{Casos prototípicos de inserción laboral}

A partir de relatos biográficos se presenta la trayectoria de los retornados cuyos casos fueron considerados prototípicos de ciertas modalidades de inserción laboral y social en Nezahualcóyotl. Estos casos fueron diseñados metodológicamente identificando factores concomitantes entre los entrevistados, y seleccionados al contrastar el conjunto de narrativas vitales recabadas mediante una entrevista semiestructurada, de corte biográfico, que permitió construir la narrativa de los llamados prototipos. ${ }^{10}$ Se construyeron dos casos: el de reinserción con reemigración y el de reinserción con establecimiento. Esta modalidad de investigación longitudinal cualitativa utiliza el análisis de las trayectorias para reconocer patrones y luego construir/elegir los casos prototípicos, que representan una tendencia o patrón de trayectorias ${ }^{11}$ que nos permite identificar los factores involucrados en el resultado del retorno internacional.

El objetivo es observar en estos casos los elementos de oportunidad ${ }^{12}$ que hacen posible la reinserción laboral y social en el contexto de retorno y cómo los actores generan estrategias de acomodamiento en esos contextos y, después, precisar la función que, en este proceso de retorno, desempeña la experiencia como inmigrante en Estados Unidos. Por último, se busca establecer el resultado del retorno en los casos seleccionados en los cuales se encuentra una intersección entre las condiciones locales y las globales de los mercados de trabajo, pero también de sociedades que funcionan de manera interconectada. A continuación se presentan los casos prototípicos de retornados en Nezahualcóyotl, que denominamos Luis y Roberto. ${ }^{13}$

Caso 1: Luis (entrevistado en febrero de 2011 en Nezahualcóyotl), 35 años, soltero, retornado de Estados Unidos a finales de 2008. Originario de Michoacán, inmigrante en Nezahualcóyotl, arribó a la edad de cuatro años. Salió de Nezahualcóyotl en enero de 2002 con destino a Nueva York a los 27 años. Era soltero, vivía en el mismo hogar que sus padres y una hermana en la colonia San

${ }^{10}$ Para una revisión amplia sobre los fundamentos y el uso de esta metodología en el análisis de trayectorias y la construcción de casos prototípicos, véase Pedreño Cánovas (2010).

${ }^{11}$ Para mayor referencia acerca del uso e implementación de la trayectoria como herramienta analítica en los estudios de migración, véase Rivera Sánchez (2012a).

${ }^{12}$ En relación con la estructura de oportunidades en el municipio, aquí sólo se delinean algunas variables a partir de las narrativas.

${ }^{13}$ Los nombres asignados a los actores que dieron pauta para construir los casos prototípicos no son los de los entrevistados. Se cambió su nombre para respetar la identidad de los informantes, quienes expresamente otorgaron el permiso para hablar de su experiencia y su vida. 
Agustín, en Nezahualcóyotl; era el mayor de tres hermanos, tenía un hermano que radicaba en la también conocida como Urbe de Hierro o la Gran Manzana y que llevaba cuatro años como inmigrante en aquella ciudad cuando Luis decidió emigrar. Es hijo de inmigrantes internos, procedentes del estado de Michoacán, sin experiencia migratoria internacional. Su nivel máximo de estudios es el segundo año de secundaria, es decir que cursó y aprobó ocho años escolares.

Luis cruzó la frontera en Nogales, Sonora, y desde Arizona viajó en avión directamente a Nueva York con el plan original de permanecer dos años para generar recursos para construir la casa paterna y solventar los gastos de la familia, no obstante tuvo una estancia de seis años continuos en Estados Unidos, esto significa que realizó un solo viaje internacional y había realizado uno de migración interna cuando era un niño. Durante su estadía en Estados Unidos vivió cinco años y nueve meses en la Gran Manzana, dos meses en Atlantic City y uno más en Pensilvania.

Cuando decidió emigrar a Nueva York trabajaba como empleado en una fábrica de embutidos en Nezahualcóyotl, donde se desempeñaba como obrero general y contaba con seguridad social (aguinaldo, seguro médico, vacaciones), ganaba un poco más del salario mínimo establecido en la zona metropolitana. Emigró solo, orientado por su hermano que estaba en Nueva York, quien le prestó dinero para hacer el viaje, el cual reembolsó en un periodo de un año $y$ medio. En los primeros 18 meses de estancia en el extranjero, no envió remesas a su familia en Nezahualcóyotl, sólo consiguió financiar su estancia y pagar el préstamo para su viaje (el cruce y el viaje por avión hasta su destino ascendieron a 28 mil pesos mexicanos). Los siguientes cuatro años y medio, Luis envió remesas mensuales a su familia establecida en Nezahualcóyotl, quienes contaban con un terreno que adquirieron previamente. Las remesas permitieron edificar la casa y avanzar en la construcción de un cuarto anexo donde él planea vivir una vez que lo concluya.

Su historia laboral inicia a los 14 años, como obrero eventual en fábricas en la zona metropolitana y como peón albañil junto con su padre, quien era maestro albañil. A los 27 años llega a Nueva York y se emplea de inmediato en la compañía en la que trabajaba su hermano, como ayudante en el manejo de materiales para la construcción de casas, por un lapso de tres años. Su segundo empleo fue en un restaurante, el cual consiguió sin el contacto de su hermano, como ayudante de cocina, pero conservó además el trabajo como ayudante de especialistas en pegar azulejo en las construcciones de casas y edificios. Tomar un segundo empleo implicaba tener una doble jornada, por la mañana en la compañía de materiales de construcción y en la tarde y noche en el restaurante, donde 
aprendió a cocinar comida rápida. Después de 18 meses de desempeñarse en ambos empleos, el restaurante cerró sus puertas y se quedó laborando en un solo empleo, pero de manera paralela tomaba trabajos temporales por una jornada o una semana, y mantenía el empleo en la companía. Los últimos ocho meses estuvo en una empresa que reparaba casas y edificios, donde ya había trabajado. Después se mudó a Atlantic City para probar suerte en otro lugar.

Durante su estancia de seis años en Estados Unidos, Luis vivió dos meses y medio en Atlantic City, donde fue contratado un mes como ayudante de cocina en un restaurante chino, $y$ un mes y medio como empleado general en una compañía de instalación de cocinas integrales.

Después de dos meses y medio y de constatar que percibía $40 \%$ menos que en la Gran Manzana, decidió volver a Nueva York. Allí se empleó durante ocho meses con un patrón puertorriqueño con quien ya había trabajado, y estuvo como empleado hasta finales de 2002 cuando decidió volver a México. Tomó la decisión al recibir un video donde sus padres le mostraron la casa casi concluida; eso lo motivó a volver, pues había alcanzado la meta propuesta. Al mismo tiempo, la muerte de una sobrina en Nueva York lo hizo reflexionar sobre el momento de su regreso. Así, tomó la determinación de volver, trayendo consigo ahorros y planeando establecer un negocio. Regresó a Nezahualcóyotl y estuvo un mes y medio sin trabajar (por decisión propia) hasta que finalmente regresó a la compañía de embutidos, en el puesto de obrero general, el mismo en el que se desempeñaba antes de emprender su viaje al extranjero.

Luis fue entrevistado en febrero de 2011, tenía tres años de haber retornado a Nezahualcóyotl, y tenía el proyecto de reemigrar a Nueva York un mes más tarde, con la intención de trabajar dos años y luego volver a Nezahualcóyotl. Contaba con los recursos económicos para financiar el viaje y planeaba llevar a un amigo primerizo (primer viaje a Estados Unidos) del municipio de Los Reyes, La Paz, un municipio vecino en la zona conurbada del Distrito Federal.

Caso 2: Roberto (entrevistado en mayo de 2012 en Nezahualcóyotl), 48 años, casado, retornado de Estados Unidos en enero de 2010, después de vivir 20 años en aquel país. Actualmente es trabajador por cuenta propia, tiene un pequeño taller de carpintería y herrería en la colonia La Perla, en Nezahualcóyotl, localizado a dos casas de la de su madre, donde residen él y su familia. Roberto es un inmigrante de Michoacán en Nezahualcóyotl, a donde llegó con sus padres a la edad de cuatro años, procedente de un pequeño pueblo. Ocupa el quinto lugar de 13 hermanos. Viajó en 1990 a California a la edad de 28 años, en concreto a Long Beach, donde vivía una hermana que estaba casada y ahí trabajaba como 
baby sitter. Roberto vivía con su hijo de cuatro años y su esposa, los tres miembros de la familia realizaron juntos el viaje.

En Nezahualcóyotl, Roberto era empleado en una fábrica, donde hacía trabajo de herrería y eventualmente también carpintería, ambos oficios los había aprendido con sus hermanos y su padre. Su motivación para emigrar no fue la falta de empleo, pues asegura que en Neza "nunca conoció la carestía de trabajo", sino un evento que lo desmoralizó: le robaron la herramienta de carpintería que había llevado a la fábrica donde trabajaba para realizar una labor especial. Decidió entonces emigrar apoyado por su hermana, quien financió el viaje a Long Beach, en octubre de 1990, pero la decisión fue tomada por Roberto y su familia en junio del mismo año, es decir que consideró cuatro meses para no abandonar de inmediato su empleo.

En los 20 años que vivió en Estados Unidos no realizó ningún viaje a Nezahualcóyotl (es decir, a México), de tal forma que en su vida ha realizado un viaje al interior del país, cuando se mudó de Michoacán a Nezahualcóyotl, y un solo viaje de México a Estados Unidos.

Roberto y su familia cruzaron la frontera en Tijuana y llegaron por tierra a Long Beach, California; vivieron con su hermana y su familia en un espacio pequeño donde habitaban al menos seis personas más, entre primos y sobrinos. Asegura que por primera vez en su vida "sentí que no podía conseguir un empleo". Llegó a principios de octubre de 1990 y consiguió su primer empleo a finales de enero de 1991, después de obtener documentos como el social security number y la green card falsos. Su primera ocupación fue como ayudante en una tintorería, en la que trabajaba su cuñado; ahí estuvo año y medio, planchando ropa. En ese tiempo pasó de ser ayudante a empleado general (planchador) cuando su cuñado llegó a ser el encargado de la tintorería. Luego de un año y medio en Long Beach se mudó al estado de Washington, muy cerca de Seattle, lugar al que se movieron los patrones de su hermana $-y$ junto con ellos su hermana $y$ toda su familia, incluidos Roberto y los suyos-. Allá fue contratado de nuevo en una tintorería, como planchador, donde permaneció siete años y medio aproximadamente. Ahí mismo trabajó su esposa hasta 1999, también por siete años, como empleada, encargada de doblar las prendas y etiquetarlas, pero nunca en la atención al público.

Roberto decidió tomar otro empleo, después de siete años y medio en la tintorería, de donde renunció por problemas de salud, debido a la exposición constante a las altas temperaturas. Se colocó como obrero en una fábrica de ventanas y aluminio, donde por sus conocimientos y habilidades adquiridas como herrero en Nezahualcóyotl rápidamente aprendió el oficio de "aluminero" 
(especialista en trabajar el aluminio). Trabajó durante 10 años en esa fábrica (1999-2009), usando siempre sus documentos apócrifos. En 2009 se enfrentó a dos dilemas vitales: $a$ ) la expiración de la tarjeta que contenía su número de seguridad social y de su green card, que le permitían ser contratado en la fábrica, no obstante que -suponía Roberto- los patrones sabían que eran falsos; $b$ ) su esposa había sido diagnosticada con una enfermedad degenerativa algunos años antes y para entonces se había agravado su condición. Además, en 2005 habían experimentado una crisis familiar que llevó a Roberto a acercarse, y más tarde afiliarse como miembro, a los testigos de Jehová, también por intermediación de su hermana, quien se había convertido a esa religión años atrás. Después de cuatro años de converso, Roberto enfrentó el dilema "moral y ético" -así lo llamade renovar sus documentos de la forma en que los había obtenido, o bien declarar en la fábrica que eran falsos y someterse a la decisión del patrón de contratarlo o no sin documentos. Tomó la segunda opción y fue despedido en los primeros meses de 2009.

Durante 2009 consiguió sólo empleos eventuales por la falta de papeles, por un día, una semana o si acaso 15 días continuos. En 2010 empeoró la condición de su esposa y, como ya no tenía seguridad social, decidió pedir ayuda a la familia de su esposa, que radicaba en Iztapalapa, Distrito Federal. Fue entonces que decidió volver con su familia a Nezahualcóyotl en diciembre de 2010.

Regresó a México con una camioneta, seis mil dólares y herramienta para realizar trabajo tanto de carpintería como de herrería; aunque la mayor parte de esa herramienta y de las técnicas aprendidas no las ha podido implementar, por ejemplo técnicas relacionadas con la fabricación de portones/zaguanes, debido a que en Nezahualcóyotl se "acostumbra" colocar portones "prácticamente blindados" en las entradas de las viviendas. Cuenta con utensilios con un valor comercial importante, como tornos y maquinaria con la que podría fabricar en serie sillas con tubulares. No obstante, optó por poner un pequeño taller de carpintería y herrería en la cochera de la casa de un hermano, a dos casas del lugar que habita. Asimismo, durante su estadía en Estados Unidos compró una casa en la periferia de Seattle, en un terreno de dos acres y medio, la cual aparentemente vendió antes de viajar de regreso a México, pero aún no ha recibido el pago.

Roberto fue entrevistado en mayo de 2012, a sólo un año y seis meses de haber retornado a Nezahualcóyotl, en donde de inmediato empezó a trabajar como carpintero y herrero. Allí se ha insertado en un Salón del Reino de los Testigos de Jehová localizado en la colonia donde reside. Ha enfrentado algunas dificultades porque lo ven "diferente" en comparación con la imagen que proyectaba en 
el momento de su partida, hace 20 años, ahora con una nueva religión y otra forma de relacionarse con los vecinos, "más respetuosa y distante" por no compartir sus festividades religiosas y cívicas. Asimismo, su madre y otros familiares -así lo afirmó Roberto- reconocen que no se integra igual en las celebraciones con la familia porque no consume alcohol, aunque hizo el esfuerzo de saludar en persona a las familias que viven en la calle donde residía antes de viajar, la misma donde ahora vive; algunos lo recordaban, otros no lo conocían. Observa muchos cambios en Nezahualcóyotl, entre otros, los lugares se han transformado, las calles ahora lucen pavimentadas, ha crecido la colonia (en términos espaciales y poblacionales, por supuesto) y se han agregado varias nuevas. Asegura que no volverá a Estados Unidos, no obstante que su sueño era regresar siendo "un inmigrante legal" y con documentos, meta que no consiguió.

Los casos se construyeron a partir de los relatos de migrantes que retornaron en los últimos cinco años; representan casos prototípicos de reinserción con reemigración, en el caso de Luis, y de reinserción con establecimiento, en el de Roberto. Estos prototipos muestran las circunstancias en las cuales ciertos eventos familiares y personales se entrecruzan y definen el momento del retorno, pero no sin estar mediado por las condiciones del mercado laboral en los lugares de destino. Asimismo, su experiencia de reinserción social y laboral es producto de la intersección entre lógicas locales/familiares y globales/trasnacionales. Es decir, lo local y lo global son entendidos como mutuamente constituyentes -recuperando la propuesta de Glick Schiller (2010)- pero difieren en escala o posición respecto del lugar que ocupan en la jerarquía de las fuerzas económicas y sociales, además de su impacto.

Por lo anterior, resulta relevante observar cuáles son las modalidades de inserción local no sólo en los lugares de destino, sino en los de retorno, debido a que la posición que ocupa la localidad en esta escala hace posible delinear diferentes vías o modalidades de inserción en la vida de esas ciudades. De manera simultánea, este ejercicio permite identificar el papel que desempeñan los habitantes con experiencia de migración interna, internacional, los nativos del lugar y, aún más, los que tienen ambas experiencias de migración, los que son trabajadores calificados respecto de los que tienen menores niveles de escolaridad $y$ calificación, en suma, observar en contexto cómo funcionan las instituciones en su relación con los habitantes del lugar, entendiendo el mercado de trabajo como una institución, puesto que opera con base en reglas y dinámicas de interconexión con los mercados de trabajo en el sistema económico global. Son estos factores los que delinean las modalidades de reinserción en el retorno y la posibilidad del 
establecimiento o la reemigración (a un destino nacional o internacional), como también sucede en el proceso de inserción (o incorporación) en el lugar de destino (cf. Glick Schiller, 2010; Glick Schiller y Caglar, 2011).

Esto significa reconocer la capacidad de transformación, de agencia, que pueden tener los migrantes en estas localidades y ciudades, más allá del efecto identificado y estudiado en la perspectiva de las ciudades globales (Sassen, 1991) o los centros metropolitanos (Glick Schiller y Caglar, 2011); en este caso, cómo Nezahualcóyotl podría reescalar su posición incluso en cuanto centro urbano de atracción para otros habitantes frente a la Ciudad de México, por ejemplo, pero no sólo como parte de un proceso de precarización, como ocurrió en sus orígenes y a lo largo de estas últimas décadas, sino posicionarse como una localidad donde la diversidad y la contribución de los inmigrantes -tanto los migrantes internos como los retornados internacionales- podrían transformar las colonias, no sólo por la construcción de edificios con nuevos diseños arquitectónicos, que se visualizan discretamente en las casas y los negocios de este municipio, sino por la capacidad para introducir otros símbolos, valores y formas de hacer $y$ concebir el trabajo, así como modalidades de convivencia en la diversidad y de otros códigos socioculturales.

\section{Comentarios finales}

En el caso del prototipo inserción con establecimiento presentado, se identifica nítidamente cómo la experiencia personal se intersecta con las condiciones históricas como inmigrante indocumentado en Estados Unidos; es decir, la enfermedad de la esposa, sumada a la falta de documentos migratorios y la decisión personal de no obtenerlos otra vez falsificados, tiene como consecuencia enfrentar una mayor dificultad para conseguir un nuevo empleo cuando es despedido de la fábrica, y luego confinarse, en la última etapa de su vida en Estados Unidos, a tomar empleos de bajo salario, corto plazo, ninguna certidumbre laboral y baja calificación, a pesar de contar con una amplia experiencia como obrero en fábricas y maquiladoras en ese país, habiendo desarrollado habilidades como herrero, carpintero y aluminero durante al menos una década de trabajo cerca de Seattle, y una década adicional en Nezahualcóyotl (antes de su viaje internacional).

Pero no sólo la decisión del retorno fue motivada por esta intersección de eventos familiares y la dinámica del contexto y las condiciones actuales de la inmigración indocumentada en Estados Unidos -la crisis de los mercados laborales que han reforzado una política aún más restrictiva en materia de derechos 
y oportunidades para los inmigrantes en el último quinquenio-, sino también la experiencia de retorno a Nezahualcóyotl y el proceso de reinserción social y laboral han sido moldeados en esta lógica. Su afiliación en 2005 a los testigos de Jehová y su involucramiento religioso durante los años posteriores influyeron en gran medida en una serie de decisiones relevantes de su vida como inmigrante, como trabajador $y$, por supuesto, como persona. El retorno es visto también - desde la perspectiva de Roberto- como el resultado de la elección de una "forma ética y moral de vida". Su reinserción social en Nezahualcóyotl no ha estado ligada a la posibilidad de reactivar las viejas redes familiares y vecinales, sino a la oportunidad de construir y activar una nueva red de relaciones a partir de los testigos de Jehová, cuyo establecimiento se localiza en la colonia donde él reside en la actualidad. Su filiación religiosa le ha permitido reinsertarse socialmente, en un lugar donde después de 20 años de ausencia se percibe a sí mismo diferente y observa cómo también han cambiado el lugar y las personas que lo habitan. En este caso, su inserción está mediada por la dinámica de las religiones globales que han alcanzado las localidades y han diversificado las opciones religiosas, que con certeza implican diferentes formas de vida y de relación con la otredad. Es decir, su conversión religiosa en Estados Unidos ha permeado su inserción social ( $y$ en algunos momentos su inserción laboral) en Nezahualcóyotl; lo mismo que su inserción laboral en Estados Unidos estuvo influida por su experiencia previa de trabajo en la carpintería y la herrería en Nezahualcóyotl.

Entonces, la lógica entre reinserción y establecimiento está mediada por la dinámica entre mercados de trabajo y modalidades de inserción social creadas a partir de la intersección de la experiencia vital e histórica de los inmigrantes en esta localidad y su vínculo con los habitantes nativos en ese espacio, sin perder de vista que ambos están atravesados por la dinámica global y la posición que ocupa la localidad en esa escala, además del contexto de retorno. Por esa razón, la experiencia de retorno urbano y rural es diferenciada, debido a la estructura de oportunidades en la que un inmigrante o retornado se inserta y a la posibilidad de transformar esas estructuras o de diseñar mecanismos que faciliten su inserción, reinserción o establecimiento, así como la reemigración a un destino internacional o a uno nacional.

En este artículo no se ha presentado la evidencia de las condiciones en términos de estructuras de oportunidad (llámense industrias, negocios, espacios de socialización y recreación, oportunidades educativas, infraestructura urbana) del municipio de Nezahualcóyotl; por ahora se han trazado las coordenadas para -a partir de analizar ciertas características demográficas en materia de ocupación y empleo de la población con experiencia de migración internacional e interna y 
que habita en el municipio- comprender algunos de esos factores que intervienen en la configuración de determinados tipos de reinserción laboral y social de migrantes retornados de Estados Unidos en contextos urbanos, y por ello la elección metodológica de presentar casos que despliegan en sus narrativas de vida los elementos que condicionan la reinserción.

Asimismo, ya que esta investigación se encuentra en proceso se optó por examinar en la primera parte cómo se acomodan algunas piezas del mercado de trabajo en Nezahualcóyotl, uno que, tanto para inmigrantes internos como para retornados contemporáneos, se concentra en categorías (situación en el empleo) como "obrero o empleado" y "trabajador por cuenta propia".

Además, el caso prototípico construido para dar cuenta de la inserción con reemigración permite delinear otras vías de inserción social con base en el hecho de que el retornado ocupa la posición de hijo en el hogar y se reinserta en la dinámica familiar del hogar paterno, y luego retoma una posición en el empleo que había ocupado antes de su viaje a Estados Unidos. ¿Cómo opera en este caso el capital acumulado y qué papel tiene en su reinserción social y laboral su experiencia de vida como inmigrante internacional en la localidad de Nezahualcóyotl, para que finalmente Luis decida reemigrar a Estados Unidos? Es decir, ¿qué factores intervinieron para que el resultado del retorno, en su caso, no fuera el establecimiento, sino la reemigración? Éstas son las preguntas que este estudio puede contribuir a responder. Por ahora se vislumbra, a través de nuestros hallazgos, la compleja condición en la que ocurre el retorno migratorio internacional en un contexto urbano metropolitano preciso en México, en el que parece que el capital humano adquirido durante la experiencia como inmigrante internacional no es la clave para reinsertarse y establecerse.

\section{Bibliografía}

Aznar, Yésica

2011 "Otro norte, otro terruño. Reconstruyendo los sentidos y las identidades de los retornados en localidades urbanas", tesis de doctorado en Ciencia Social con especialidad en Sociología, Centro de Estudios Sociológicos-El Colegio de México (Colmex), México.

Basch, Linda, Nina Glick Schiller y Christina Szanton Blanc

1994 Nations Unbound: Transnational Projects, Postcolonial Predicaments, and Deterritorialized Nation-States, Gordon y Breach, Langhorne.

Canales, Alejandro

1999 "Periodicidad, estacionalidad, duración y retorno. Los distintos tiempos 
en la migración México-Estados Unidos", en Papeles de Población, núm. 22, octubre-diciembre, pp. 11-41.

Cassarino, Jean-Pierre

2004 "Theorising Return Migration: The Conceptual Approach to Return Migrants Revisited", en International Journal on Multicultural Societies, vol. 6 , núm. 2, pp. 253-279.

Durand, Jorge

2004 "Ensayo teórico sobre la migración de retorno. El principio del rendimiento decreciente", en Cuadernos Geográficos, núm. 35, pp. 103-116.

2009 "La situación actual de los mercados de trabajo en Estados Unidos y las dinámicas contemporáneas de la migración mexicana“, conferencia magistral en el Seminario Permanente de Migración de El Colegio de la Frontera Norte, sede Tijuana, 13 de marzo.

Durand, Jorge y Douglas Massey

2003 Clandestinos. Migración México-Estados Unidos en los albores del siglo XXI, Miguel Ángel Porrúa/Universidad Autónoma de Zacatecas, México.

Espinosa, Víctor M.

1998 El dilema del retorno, género y pertenencia en un contexto transnacional, El Colegio de Jalisco/El Colegio de Michoacán, México.

Glick Schiller, Nina

2007 Beyond the Nation-State and its Units of Analysis: Towards a New Research Agenda for Migration Studies. Essentials of Migration Theory, Center on Migration, Citizenship and Development (Working Paper, núm. 33), Bielefeld.

2010 "A Global Perspective on Migration and Development", en Nina Glick Schiller y Thomas Faist, Migration, Development and Transnationalization. A Critical Stance, Berghahn Books, Oxford, pp. 23-62.

Glick Schiller, Nina y Ayse Caglar

2007 "Beyond Methodological Ethnicity and Towards City Scale. An Alternative Approach to Local and Transnational Pathways of Migrant Incorporation", ponencia presentada en la Conference on Transnational Migration and Development, Bielefeld, 30 de mayo a $1^{\circ}$ de junio.

Glick Schiller, Nina y Ayse Caglar (eds.)

2011 Locating Migration, Rescaling Cities and Migrants, Cornell University Press, Ithaca y Nueva York.

Herrera, Gioconda et al.

2013 "El retorno a Ecuador desde España: Estrategias económicas, socio-familiares y afectivas para una integración transnacional”, informe de investigación, Facultad Latinoamericana de Ciencias Sociales (Flacso), Quito. 
Ibarra Templos, Yuribi

2011 "Migración indígena de retorno a Ciudad Nezahualcóyotl, ¿La etnicidad como elemento de diferenciación en la reinserción social?", proyecto de investigación posdoctoral, marzo de 2011 a febrero de 2012, Centro Regional de Investigaciones Multidisciplinarias-Universidad Nacional Autónoma de México (UNAM), México.

INEGI

2000 Censo General de Población y Vivienda, 2000, Instituto Nacional de Estadística, Geografía e Informática, México.

2005 Conteo de Población y Vivienda, 2005, Instituto Nacional de Estadística, Geografía e Informática, México.

2010 Censo General de Población y Vivienda, 2010, Instituto Nacional de Estadística y Geografía, México.

2012 Boletín de Prensa, núm. 204/12, Instituto Nacional de Estadística y Geografía, México, 5 de junio.

Lindón, Alicia

1999 De la trama de la cotidianidad a los modos de vida urbanos. El Valle de Chalco, Colmex/El Colegio Mexiquense, México.

Massey, Douglas et al.

1987 Return to Aztlan: The Social Process of International Migration from Western Mexico, University of California Press, Berkeley.

Papail, Jean

2002 "De asalariado a empresario: La reinserción laboral de los migrantes internacionales en la región centro-occidente de México", en Migraciones Internacionales, vol. 1, núm. 33, julio-diciembre, pp. 79-102.

2005 "Remesas e inversiones de los migrantes de retorno en el centro-occidente de México", en Raúl Delgado Wise y Beatriz Knerr (coords.), Contribuciones al análisis de la migración internacional y el desarrollo regional en México, Miguel Ángel Porrúa/Cámara de Diputados/Universidad Autónoma de Zacatecas, México, pp. 319-332.

Pedreño Cánovas, Andrés

2010 "Familias inmigrantes: el trabajo de los padres y las estrategias de trabajo de los hijos en las áreas mediterráneas de agricultura intensiva", en Sara Lara Flores (coord.), Migraciones de trabajo y movilidad territorial, Miguel Ángel Porrúa, México, pp. 333-365.

Rivera Sánchez, Liliana

2008 "El eslabón urbano en el trayecto interno del circuito migratorio MixtecaNueva York-Mixteca: Los migrantes de Nezahualcóyotl, Estado de México", 
en Elaine Levine (ed.), La migración y los latinos en Estados Unidos. Visiones y conexiones, Centro de Investigaciones sobre América del Norte-UNAM, México, pp. 53-73.

2011 ¿Quiénes son los retornados? Apuntes sobre el migrante retornado en el México contemporáneo", en Bela Feldman-Bianco et al. (comps.), La construcción social del sujeto migrante en América Latina. Prácticas, representaciones y categorías, Flacso/Consejo Latinoamericano de Ciencias Sociales (Clacso)/ Universidad Alberto Hurtado de Chile, Quito, pp. 309-338.

2012a "Las trayectorias en los estudios de migración: una herramienta para el análisis longitudinal cualitativo", en Marina Ariza y Laura Velasco, Métodos cualitativos y su aplicación empírica. Por los caminos de la investigación sobre migración internacional, Instituto de Investigaciones Sociales-UNAM/El Colegio de la Frontera Norte, México, pp. 455-494.

2012b Vinculos y prácticas de interconexión en un circuito migratorio entre México y Nueva York, Clacso, Buenos Aires.

Rivera Sánchez, Liliana y Fernando Lozano Ascencio

2006 "Los contextos de salida urbanos y rurales y la organización social de la migración”, en Migración y Desarrollo, núm. 6, primer semestre, pp. 45-78.

Sassen, Saskia

1991 The Global City. New York, London, Tokyo, Princeton University Press, Princeton.

Secretaría de Gobernación

1999 Enciclopedia de los municipios de México: Nezahualcóyotl, Estado de México, Secretaría de Gobernación, México.

Villa, Marta Inés

2013 "Retorno de desplazados en el eje cafetalero: el caso de San Carlos en Medellín, Colombia. Una mirada desde la historia oral y la memoria", ponencia presentada en la VI Reunión del Grupo de Trabajo "Migración, Cultura y Políticas" del Clacso, Ciudad de Heredia, 4 a 6 de junio. 
Liliana Rivera Sánchez

Doctora en Sociología por The New School for Social Research, Nueva York. Sus áreas de interés son la relación entre procesos migratorios y diversificación religiosa, el retorno migratorio contemporáneo en contextos urbanos, y la práctica de la investigación en migraciones y movilidades (metodologías, métodos y herramientas). Se ha desempeñado como investigadora en el Centro Regional de Investigaciones Multidisciplinarias (CRIM) de la UNAm durante nueve años. Actualmente es profesora-investigadora en el Centro de Estudios Sociológicos de El Colegio de México. Algunas de sus publicaciones más recientes son: Vínculos y prácticas de interconexión en un circuito migratorio entre México y Nueva York (Clacso, 2012); "Las trayectorias en los estudios de migración: una herramienta para el análisis longitudinal cualitativo", en Marina Ariza y Laura Velasco (coords.), Métodos cualitativos y su aplicación empírica: por los caminos de la investigación sobre migración internacional (IIs-Unam/Colef, 20I2); y coeditora de The Practice of Research on Migration and Mobilities (CRIM-UnAm/Springer, 20I4).

Citar como: Liliana Rivera Sánchez (2013), "Reinserción social y laboral de inmigrantes retornados de Estados Unidos en un contexto urbano", Iztapalapa. Revista de Ciencias Sociales y Humanidades, núm. 75, año 34, julio-diciembre de 2013, ISSN: 2007-9176; pp. 29-57. Disponible en <http:// revistaiztapalapa.izt.uam.mx/index.php/izt/issue/archive >. 